\title{
Evaluating the Effects of Randomness on Missing Data in ArChaeological NETWORKS
}

\author{
Robert J. Bischoff \\ Cecilia Padilla-Iglesias \\ Claudine Gravel-Miguel \\ Arizona State University \\ University of Zurich \\ Arizona State University \\ rbischoff@asu.edu
}

\begin{abstract}
Network science shows promise for archaeologists who want to explore past social dynamics using material culture. Yet, archaeological data is subject to important caveats that exist for all datasets. Almost all archaeological datasets are biased, and these biases are often unknown or only partially understood. Prior research has examined the effects of missing nodes on archaeological networks. Here, we instead focus on the impact of missing links on such networks. We used an agent-based model (ArchMatNet) to generate a simulated, unbiased assemblage of artifacts deposited at sites. We link those sites through the similarity of their artifacts to form the complete network. We also include an obsidian dataset from the US Southwest to compare differences between real and simulated data. We explore how random and nonrandom sampling of the two datasets affect the accuracy of the network reconstructed. Our analysis confirms prior research demonstrating that random samples are representative of the original network, even when they are small, but that biased samples of any size are significantly problematic. This research highlights the need to consider bias in archaeological data and demonstrates the utility of agent-based models in testing archaeological methods. Furthermore, this simulated dataset can better inform how archaeologists judge bias and will help us develop new methods to mitigate the effects of biased data.
\end{abstract}

Keywords: agent-based model, social networks, sampling bias

\section{Introduction}

In the past few decades, research has accumulated showing that cultural transmission has played an important role in modern human evolution (Boyd and Richerson 1985; Greenbaum et al. 2019; McBrearty and Brooks 2000; Migliano et al. 2020). With this in mind, archaeologists have been experimenting with different methods to study past social networks as the channels through which such cultural transmission would have taken place (Gravel-Miguel and Coward n.d.). To date, most researchers studying the cultural dynamics between past populations use similarities in archaeological assemblages to reconstruct the latent social networks that produced them (e.g., Birch and Hart
2018; Golitko et al. 2012; Mackay, Stewart and Chase 2014; Mills et al. 2013; Peeples and Haas 2013; Stiner and Kuhn 1992). However, not all studies incorporated formal network analyses to their methods. With a few exceptions, the application of network structure and analysis methods to archaeological datasets made little impact outside of Oceania for some time (see Hage 1977; Hage, Harary and Others 1991; Hunt 1988; Irwin 1978; Knappett 2008; Terrell 1976, 1977, 2010). Nonetheless, this type of research has recently seen a significant increase in popularity (Brughmans and Peeples 2017; Collar et al. 2015).

Formal Social Network Analysis (SNA) emphasizes the relationships among nodes (e.g., individuals, households, settlements, even objects), rather than 
the nodal attributes traditionally studied by archaeologists (e.g., status of individuals and function or size of settlements or objects). Therefore, these methods are particularly well suited for regional analyses where settlements are treated as nodes (e.g., Mills et al. 2013). The basic principle of using SNA to evaluate patterns in archaeological assemblages is that similarities in the objects found at two sites can be used to assess the strength of social connections among those sites, whether direct or indirect, material or informational (see Mills et al. 2013; Peeples et al. 2016). Such similarities can be driven by a number of social processes including exchange, emulation, population movement, and especially, shared contexts of production or learning.

SNA can provide important information on social systems, especially when applied to "big data" (for recent overviews of SNA in archaeology, see Brughmans 2013; Brughmans and Peeples 2017; Mills 2017; Peeples 2019). Yet, applying it to archaeological datasets can be difficult, because the archaeological record represents only a subset of the material remains of past populations. This presents a problem, not only because it leads to reduced sample sizes, but also because the material remains that are now missing from the archaeological record are rarely random (Clarke 1973). In other fields, problems that arise from nonrandom missing data can be largely mitigated by designing an appropriate sampling strategy. However, this is not as easily done for archaeology, because archaeological datasets are affected by multiple different biases that affect the probability that material remains will be recovered in different places. Most importantly, archaeologists often do not know which biases affect their datasets, which may derive from the geographical location of sites, differences in excavation procedures, the selective preservation of certain sites or parts of sites due to geomorphological processes, or a combination of them (Fig.1). These biases create nonrandom samples that may be unrepresentative of the studied population, which is a well-known and sometimes intractable problem in archaeology. This problem affects all aspects of archaeologists' analyses of their dataset.

Despite the apparent unsuitability of biased archaeological datasets for statistical tests, given their violation of the assumption that one is working with random samples, these can still provide useful and robust results if archaeologists take these biases into explicit consideration (Drennan 2009). For example, samples that share the same bias can be compared. They may not represent the general population, but some conclusions can still be drawn. Also, if the bias is known, statistical differences may still be meaningful if the results contradict the biases. This second point is a judgement call. For instance, a haphazard surface collection of ceramics may bias large sherds with visible designs, resulting in a sample with $60 \%$ of the sherds decorated. In this case, it would be rash to say that this assemblage indicates a preference for decorated sherds, given the lack of sampling control. However, one could safely assume that the excavated collection contains relatively more decorated sherds in an assemblage carefully excavated to avoid bias where $90 \%$ of the sherds are decorated. In practice, detecting and accounting for sampling bias is not an either-or scenario. Often we must judge the relative representativeness of the sample. Thus, rather than asking 'is it unbiased?', the question may become 

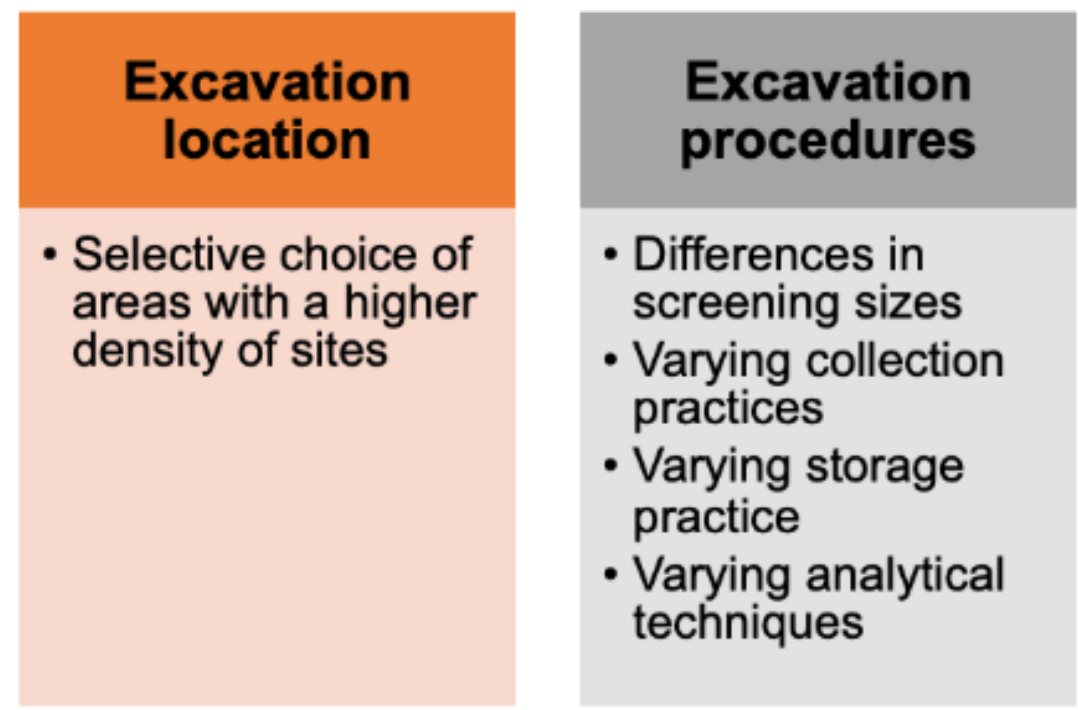

\section{Selective preservation}

- Erosion can remove or bury parts of sites

- Different soil preservation properties

Figure 1. Different sources of bias in archaeological datasets.

'is it too biased?' However, to make informed decisions, archaeologists need a better understanding of how nonrandom or biased samples affect archaeological interpretations. Here, we provide information on this topic by focusing on the impact of nonrandom missing data on archaeological networks.

\section{1.a Missing Data and}

\section{Archaeological Networks}

Studying the impact of missing data on social networks is not a new area of research. A large and robust body of literature have already provided valuable tools to assess the effects of sampling and missing information on different kinds of network properties (Bolland 1988; Borgatti, Carley and Krackhardt 2006; Costenbader and Valente 2003; Galaskiewicz 1991; Gjesfjeld 2015; Lee, Yook and Kim 2009; Rivera-Hutinel et al. 2012). From these emerged a popular method of assessing the robustness of different structural and positional aspects of a network, which is to take an existing network sampled from a (perhaps unknowable) population, resample it multiple times, and analyse the network metrics of those subsamples. Such a procedure allows estimating the degree to which certain aspects of a sampled network approximate the same aspects of the original "complete" network from which it was drawn. Using this method, Costenbader and Valente (2003) showed that if a particular metric is robust to sub-sampling, we can be confident that it approximates the value it would have in the complete network. Moreover, Costenbader and Valente found that the degree and eigenvector centrality metrics are both quite robust to node sub-sampling for both weighted and binary networks, whereas the betweenness metric changes considerably from the original sample as the sampling fraction decreases for both network types. The robustness of the former two metrics is perhaps not surprising since these measures rely on first-order connections.

Using a similar approach, Peeples and colleagues (2016) evaluated the impact of missing data on networks built from ceramic wares from the US Southwest. In this research, the authors varied the method slightly. They first created 
networks from the ware dataset at 50 year internals. For each reconstructed network, they then randomly subsampled sites with sampling fractions between $10 \%$ and $90 \%$ (at $10 \%$ intervals) of the total number of sites and evaluated the characteristics of the sampled network. Through this systematic research, they not only confirmed Costenbader and Valente's (2003) results that both degree and eigenvector centrality metrics correlate very well between the complete and sub-sampled networks, but more importantly, they also found that this high correlation persists even with sampling fractions as small as $10 \%$ of the complete site population. Moreover, the fact that they tested different sample sizes on networks pulled from different 50-year increments allowed them to show that time periods with fewer sites tend to show greater variability in centrality measures' correlations at low sample fractions. Therefore, this indicates that many network measures are robust to missing data, but small sample sizes (the number of nodes in the network) should be treated cautiously.

While resampling networks is an effective and recommended method to evaluate the impact of missing nodes on reconstructed networks, it does not touch on the impact of missing data one would use to create the links between documented nodes. This problem is specific to archaeological networks created using shared types of material culture; missing objects can lead to an underrepresentation of shared types between sites. Moreover, as with the examples discussed above, it is not possible to directly measure the effects of missing data if we do not have the full network as a source of comparison. Hence, when trying to assess the impact of missing data on our reconstructions of archaeological networks, we can instead rely on simulated datasets that share properties with those of analytical interest. Such a method allows creating an artificial complete dataset of interactions and cultural transmission. We can use this dataset to reconstruct the "complete" network and compare it to networks reconstructed from the dataset subsampled in different biased ways. This is what we present here.

When reconstructing the past from archaeological assemblages, we are always dealing with probabilities of errors, regardless of the method we use to account for biases. Rarely are we certain of our interpretation of the past, and while perfect network reconstructions will probably never be attainable in archaeology, we need to better understand how badly systematic bias and nonrandom sampling can affect our network reconstructions to help us know where to direct our efforts when conducting network analysis. Because archaeological datasets are often so problematic, it is useful to compare how nonrandom sampling can affect our interpretations by utilizing a dataset free of bias to ensure a valid comparison. Therefore, to estimate the effects of missing data on reconstructed networks, here we use data created from an agent-based model (ABM) designed to simulate interactions and material culture networks. The model creates simulated archaeological assemblages, which we sample in different ways and use to create network reconstructions. The simulated network data provides the means to test the impact of random and nonrandom sampling methods; because we have the full population of simulated data, there is no sampling bias in the initial dataset, unlike most archaeological datasets.

ABMs are excellent tools for testing archaeological methods and theories, 

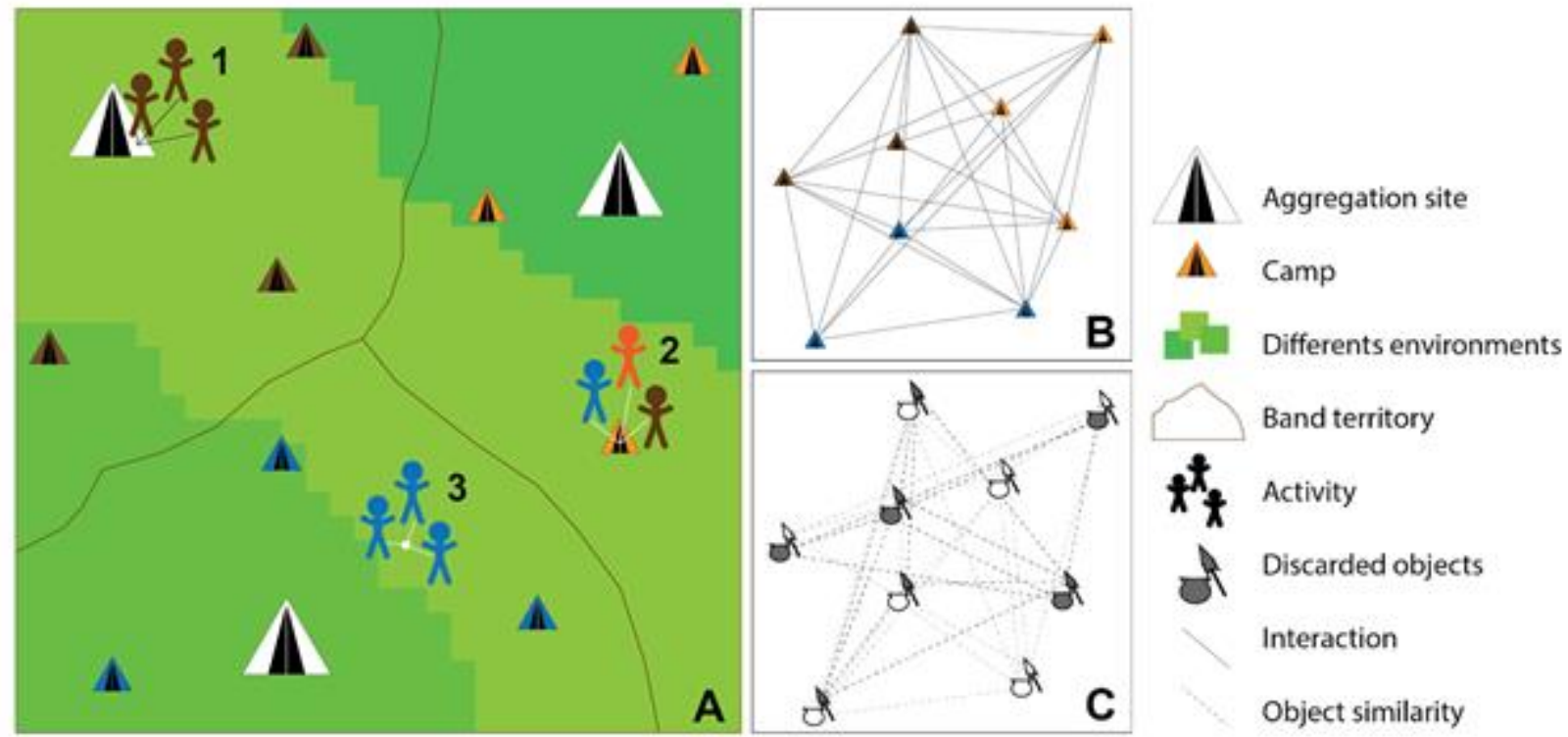

Figure 2. A. Simplified representation of the ArchMatNet model, showing the population distribution within the environment and band territories, as well as the possible activities taking place at any time step: 1. Aggregation, 2. Visit of allied agents, and 3. Hunt. B and C. Outputs created by the model: B. inter-camp interactions, and C. location and similarities of objects discarded at camps.

but they cannot replace real data. Therefore, to complement the results obtained from the dataset created by the ABM, we also use obsidian data from the U.S. Southwest. To our knowledge, no one has yet studied the effects of missing or biased data on network reconstruction in that particular dataset. Therefore, this seems like the perfect complement to our ABM data to evaluate the impact of nonrandom sampling on reconstructions of archaeological networks.

\section{Materials}

For this project, we apply the same process to two different datasets. The first dataset is created by an ABM, whereas the second is an archaeological dataset that comes from the Roosevelt Platform Mound Study.
Both the sources and the formats of the two datasets are described below.

\section{ArchMatNet}

Here we use the ArchMatNet model (or Archaeological Material Networks). It is written in NetLogo 6.2.0. (Wilenski 1999) and represents a simplified socioecological setting composed of a few groups of hunter-gatherers (henceforth referred to as 'agents') (see Fig. 2). It is an abstract model that is not meant to represent any particular area, time period, or culture. Instead, it includes multiple parameters that can be changed by the user to create different scenarios or to match particular regional ethnographic settings and/or archaeological time periods.

In this model, the population of agents is divided equally between camps that 

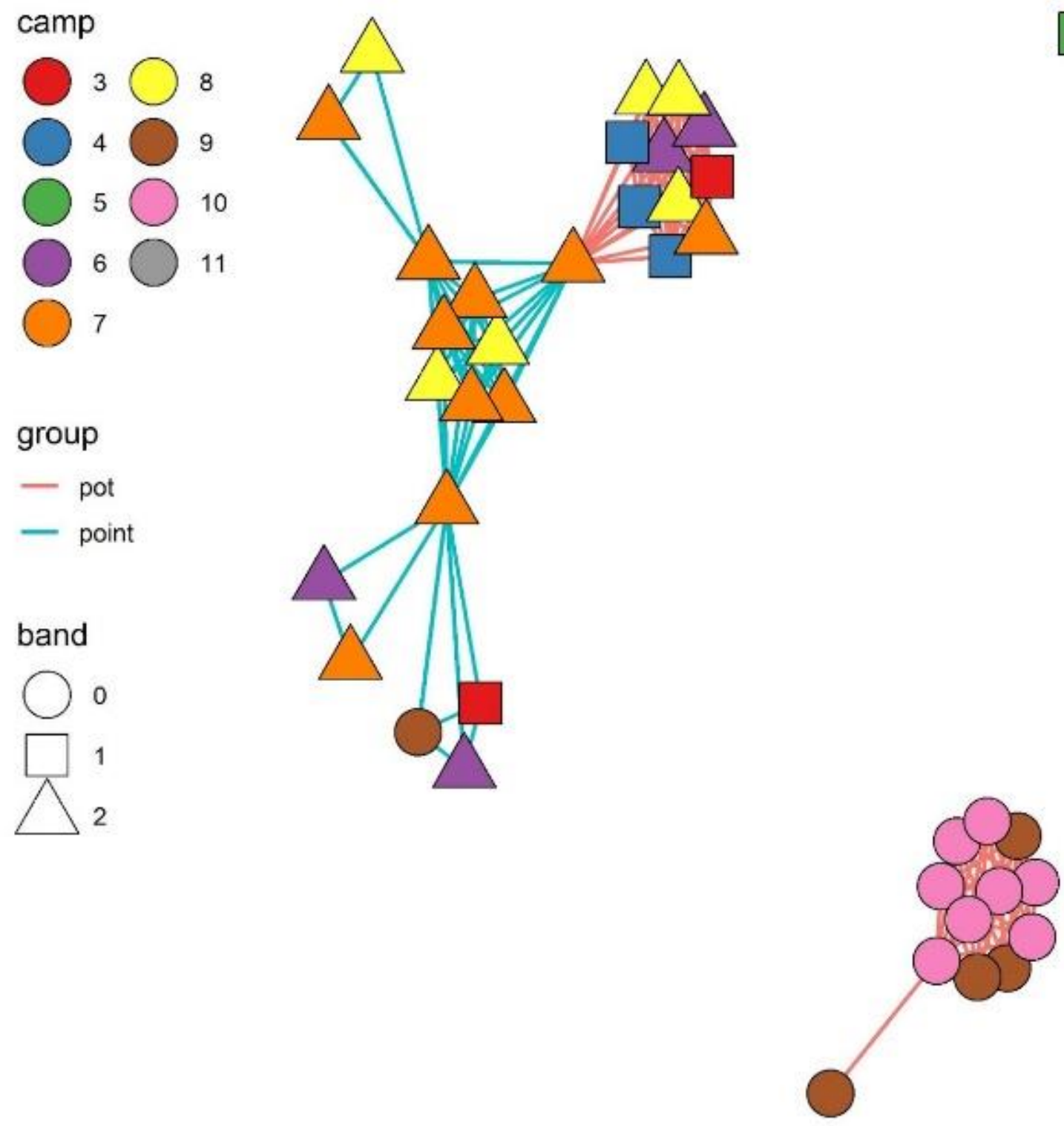

Figure 3. Network graph showing links between agents in the ArchMatNet model based on whether the agents share the same pot or point type. In this example, there is little crossover between pot and point types. Network clusters often represent the same band, although the point types are more densely connected than the pot networks. 
belong to specific bands. Each band contains three camps, but the number of agents per camp can be changed by the user. Agents make and own two sets of objects: hunting tools and ceramic pots (set to represent utilitarian and non-utilitarian objects, respectively).

Agents can own ten specimens of each object. Agents create objects based on types they know. types are modeled as integers and can have variants represented by a letter following the integer (e.g., 1a or 3c are variants on type 1 and 3, respectively). Agents can invent new types and variants during a simulation, and there are no limits set to the number of types that can be invented. Agents can learn types and variants from other agents with whom they interact (see Fig. 3).

The model is set on a simplified landscape that can vary either through time or spatially. The landscape itself does not have any resources, but the zones in which agents hunt affect their hunting success as the fitness of each hunting tool type varies depending on the environment in which the hunt takes place. In turn, an agent's hunting success can affect the likelihood that its type will be copied by other agents.

Each time step in the simulation represents one month, during which the agents can participate in a set of different activities: they can form alliances with other agents, visit allies, trade objects with nearby agents, hunt with agents from their band, and create objects based on the types they know. At regular intervals, all agents aggregate with the members of their own band. The frequency and duration of the aggregations are set by the user. Every once in a while, two agents from different camps change places to represent a simplified migration pattern. With each interaction, agents have a chance to learn from each other or to invent new types of tools. Each interaction is recorded at the individual and camp level. Figure 4 demonstrates the networks of interaction created during a model run. Interactions are designed to be frequent between camps of the same band, but interaction with other bands occurs less often. Agents do not get old or die, but over time, they can lose some knowledge, lose alliances, and drop objects on the ground. Each site contains a record of all of the objects dropped on that site, including the time-step at which they were created as well as their type. These discarded objects create the archaeological assemblage. The model has been archived through CoMSES (Janssen et al. 2008 see data availability statement).

The model offers lots of flexibility, including in the type of outputs it can produce. For this research, our focus was not to see if/how we can use discarded objects to reconstruct networks of interactions between agents. Rather, we wanted to use it to create an unbiased dataset of discarded objects to explore how sampling such a dataset would affect what it could tell us about networks. Therefore, the outputs we used here are a list of all pots discarded at sites throughout only one simulation. This list includes the name of the site where the pots were discarded, as well as the 'age' (timestep when they were created), and type of each discarded pot. 
visiting

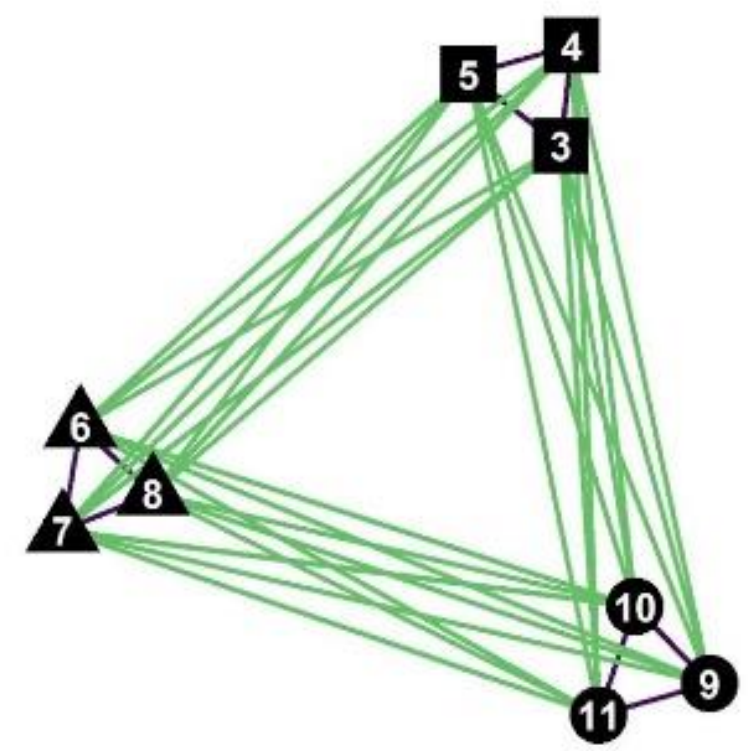

trading
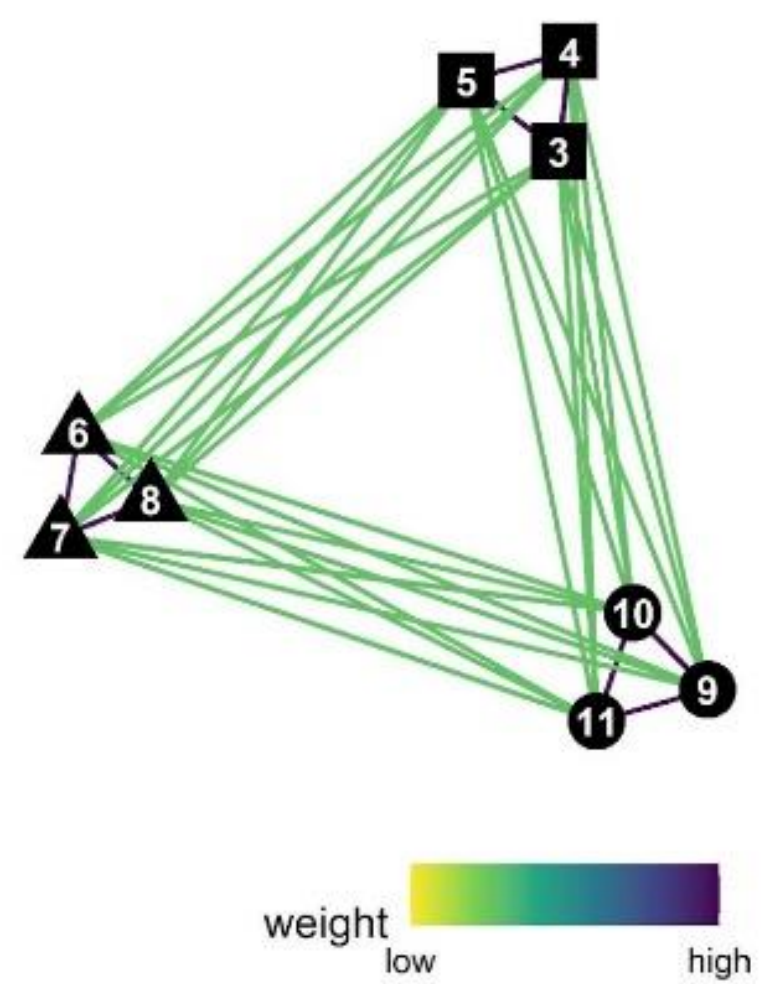

hunting

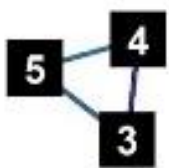

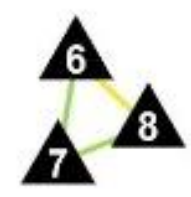

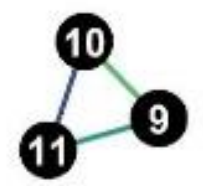

learning

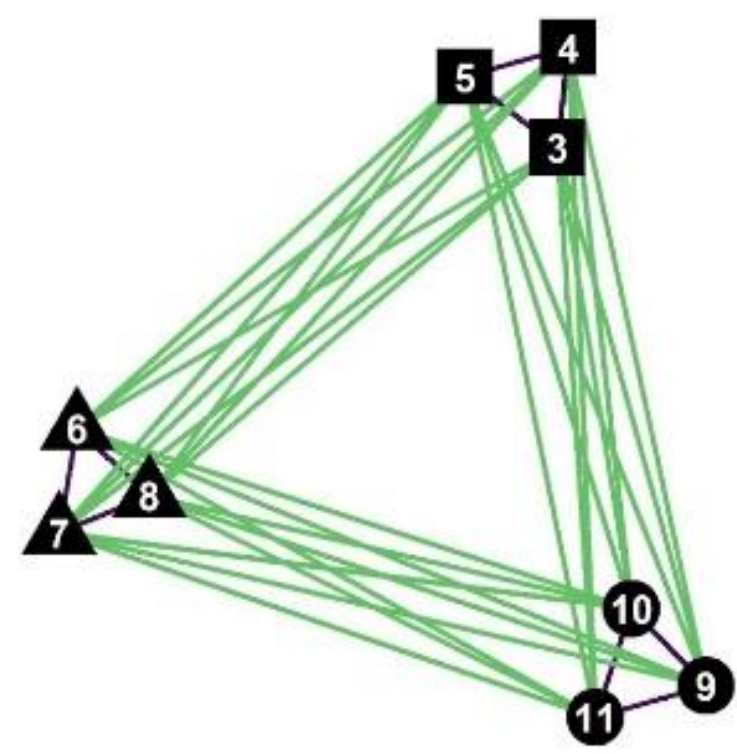

band
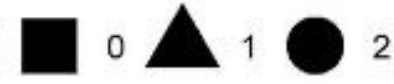

Figure 4. These networks show the interactions between each camp for every type of activity. The links are weighted by the number of interactions that occurred between agents in each camp throughout the ABM. ArchMatNet is designed to encourage most interaction to occur within bands, but each camp usually interacts in some way with each other camp. Because hunting is restricted to camps within the same band, there are no interactions shown between bands for hunting. 


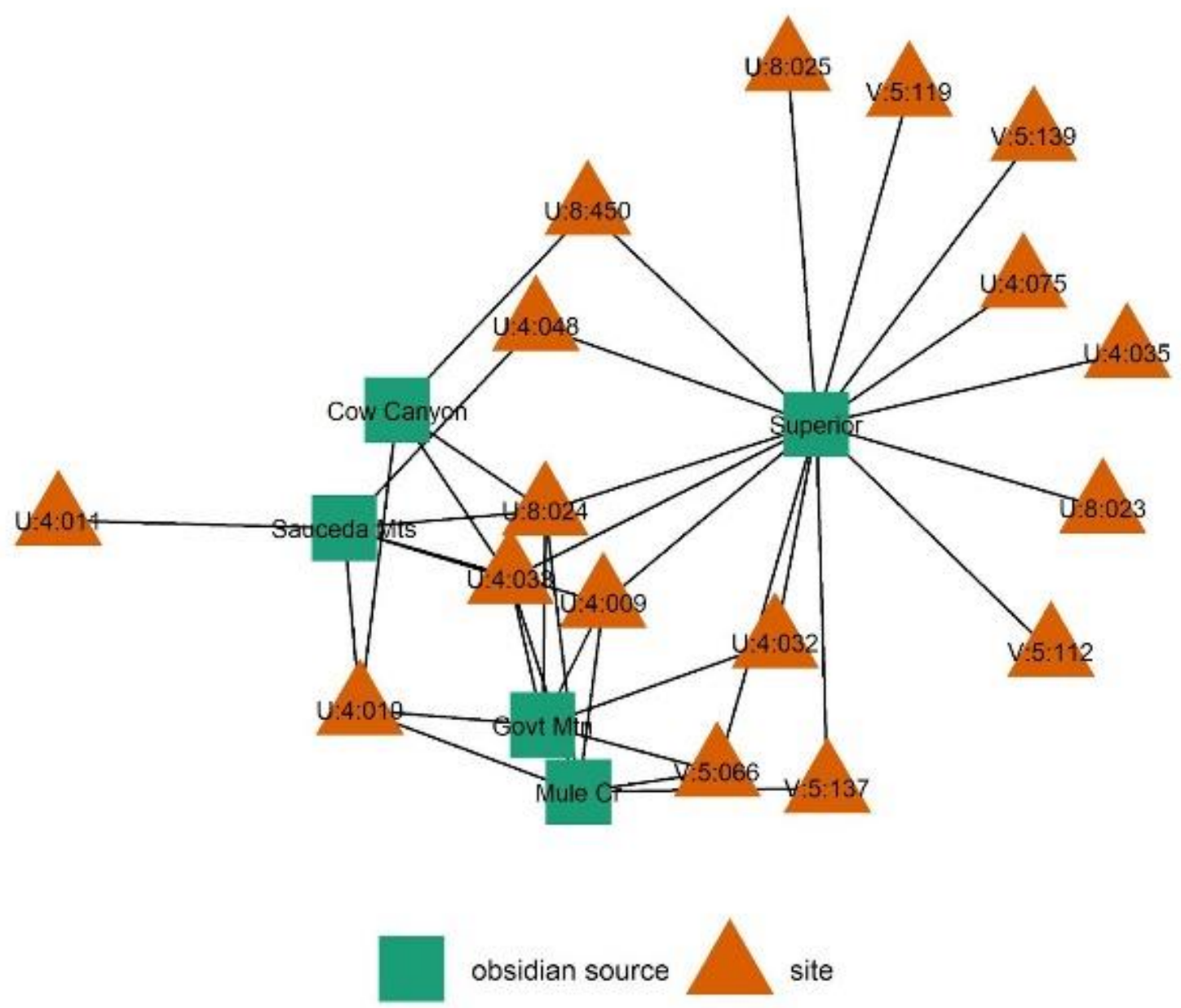

Figure 5. Bipartite presence/absence network showing which obsidian sources are found at each site in the case study from the Roosevelt Platform Mound Study. Bipartite networks only connect nodes of different types (sites only connect to obsidian sources).

\section{Obsidian Artifacts}

In addition to the ABM, we use data on sourced obsidian artifacts from the Roosevelt Platform Mound Study, which was a large cultural resource management project led by Arizona State University that took place from 1989 to 1998 in central Arizona in the U.S. Southwest (Rice 1998). This dataset contains a total of 171 sourced obsidian artifacts from 17 sites with 5 different sources of obsidian ( see Fig. 5). This dataset includes the archaeology site where each obsidian artifact was found, the specific feature that each piece of obsidian came from within the site, as well as the geological source of that obsidian (Roosevelt Platform Mound Study (RPMS) 2014).

\section{Analytical Methods}

\section{5.a Material Networks}

Defining networks in archaeology involves a process of abstraction and representation where entities (i.e., a site) become nodes and a relationship (i.e., artifact similarity) becomes a tie 
between nodes (Brandes et al. 2013: 2). A network based on material culture is not equivalent to a typical social network, such as a friendship network, and these networks are often called "material networks."

We created a material network from the output of one model run of ArchMatNet (see supplementary material for model parameters-see data availability statement). Each camp site formed a node and the pot assemblages recorded for each camp site were used to create artifact similarity matrices. For each assemblage, we calculated the proportion of each pot type. This data was then converted to an adjacency matrix showing the weighted Jaccard similarity (Marczewski and Steinhaus 1958) between each paired sites' assemblage. Weighted Jaccard similarity is useful to compare the similarity between samples and accounts for the proportion of objects in each assemblage. An adjacency matrix can easily be converted into a network graph, but many network statistics can be calculated directly on the matrix itself.

To create the obsidian material networks, we followed the same procedure, but used the obsidian source to tabulate data instead of the pot type. Nodes represent each archaeological site, and the tie between each node was also defined by the weighted Jaccard similarity between sites.

\section{5.b Random vs Nonrandom}

\section{Sampling}

To evaluate the accuracy of archaeological network reconstructions, where raw data likely result from already nonrandom sampling, we took multiple samples of the two datasets using different proportions of the total data. For the ABM output, we took samples covering from $0.1 \%$ to $90.1 \%$ of the total dataset (by 10\% increments), whereas we sampled $25 \%$, $50 \%$, and $75 \%$ of the obsidian dataset. Each dataset was sampled 1000 times for each proportion size and type of sample (nonrandom vs random). This resulted in 20,000 total samples of the ABM data and 6,000 total samples of the obsidian data.

For both datasets, the random sampling was done by taking an equal proportion of artifacts from each site. This method, also called a stratified sample design, ensured that each site (or node in the network) existed in each sample.

The nonrandom sample procedure was designed to represent gaps where time periods were absent or underrepresented in the data. This creates an artificial bias that could mimic taphonomic processes that destroyed artifacts of a certain age. The procedures differed somewhat for the $\mathrm{ABM}$ and obsidian data. We sorted the ABM dataset by the tick each pot was created, which led to a table of artifacts in order of age meant to represent the deposition of artifacts over time. We grouped the obsidian artifacts by the feature they were excavated from, which are assumed to be of similar deposition age. For both datasets, we then chose random starting points in the dataset and extracted a continuous sample starting at that point. For example, the sorted ABM dataset was split by each camp, and a random starting row was picked. All rows below the starting row were collected in the sample, up to the appropriate sample size. If the sample size was too small (e.g., when the starting point was very close to the end of the simulation, or close to the youngest archaeological feature with obsidian artifacts), we complemented it by going back to the first row and include it as well as 
following ones until we reached the target sample size. Because the types of tools change through time during a simulation, this can end up removing a critical time period where two sites were interacting more or less frequently than at other time periods (see supplementary material for further details - see data availability statement).

For each sampled dataset, we computed new Weighted Jaccard adjacency matrices using the methods detailed above to reconstruct the network. We then used the NetworkDistance package (You 2020) in $R$ ( $R$ Core Team 2021) to compute the Laplacian discrete spectral distance (Chung 1997) between these networks and the networks reconstructed from the complete dataset. We chose to use the Laplacian metric for these comparisons, because it has proven more effective than similar methods at comparing networks (Shimada et al. 2016; Tantardini et al. 2019; Wills and Meyer 2020).

\section{Results}

\section{6.a ArchMatNet}

The results of the ArchMatNet analysis suggest that nonrandom sampling of datasets lead to inaccurate network reconstructions, but that random samples do not. This is visually apparent in Figure 6, which shows a comparison between the networks reconstructed from the complete simulated dataset and from a $20 \%$ sample taken randomly and another taken nonrandomly. These networks were created using the weighted Jaccard similarity matrices. The assemblages represented in this figure are an aggregate of all pots discarded at each camp site, including agents visiting from different camps. The 50th percentile of the weighted Jaccard similarity was used as a cutoff for ties shown in the graph, so that only the strongest ties are visualized. This shows the different impacts random and nonrandom samples have on our reconstructions; even at $20 \%$ of the data, the randomly sampled network is almost identical to the original network, whereas the network made from a nonrandomly sampled network is much different. With the nonrandom sample, the strength of ties vary considerably and many ties are missing. Notably, camp 10 no longer has visible ties to any other nodes, which suggests that its connections with other sites are weaker based on this subsample than when considering the complete dataset.

The impact of sample size on network reconstruction accuracy for both types of samples is shown in Figure 7. Only extremely small random samples of $0.1 \%$ of the total data varied significantly from the original network. Each of the sample sizes had a significantly lower mean Laplacian distance than the nonrandom samples (Table 1; $p$-values represent a onetailed t-test testing whether nonrandom samples have a lower mean than random samples). Even a 10\% random sample size of this data produced a network that closely resembled the original network. Thus, when taken from an unbiased and complete dataset, random sampling should produce accurate network results, with the exception of extremely small samples.

Unfortunately for archaeologists, the nonrandom samples tell a different story. Although larger samples are better, even a sample of $90 \%$ of the full dataset taken nonrandomly produces a greater Laplacian distance than the random sample of $10 \%$ of the dataset. Of the 9000 nonrandom samples between $10-90 \%$, only one sample (with a $10 \%$ sample size) had a smaller 
Table 1. ABM Laplacian Distances by Sample Size and Type

\begin{tabular}{lllll}
\hline sample proportion & sample size & random mean & nonrandom mean & p-value \\
\hline $0.1 \%$ & 234 & 2.03 & 4.86 & $<0.001$ \\
$10.1 \%$ & 24,200 & 0.143 & 3.95 & $<0.001$ \\
$20.1 \%$ & 48,100 & 0.098 & 3.31 & $<0.001$ \\
$30.1 \%$ & 72,000 & 0.073 & 2.81 & $<0.001$ \\
$40.1 \%$ & 95,900 & 0.058 & 2.36 & 0 \\
$50.1 \%$ & 120,000 & 0.047 & 1.95 & 0 \\
$60.1 \%$ & 144,000 & 0.039 & 1.51 & 0 \\
$70.1 \%$ & 168,000 & 0.031 & 1.09 & 0 \\
$80.1 \%$ & 192,000 & 0.024 & 0.725 & 0 \\
$90.1 \%$ & 216,000 & 0.016 & 0.382 & \\
\hline
\end{tabular}

Laplacian distance than the mean distance for the randomly sampled networks. The mean Laplacian distance for nonrandom samples at a $90 \%$ size was almost three times higher than the $10 \%$ sample size taken randomly. Therefore, the results of these tests show that the disparity between nonrandom and random samples is large enough that it cannot, or at least should not, be ignored. Many of us feel safer dealing with sampling bias when we have a large amount of data.

However, this study indicates that even large samples can be heavily affected by biases in the sample. 
all

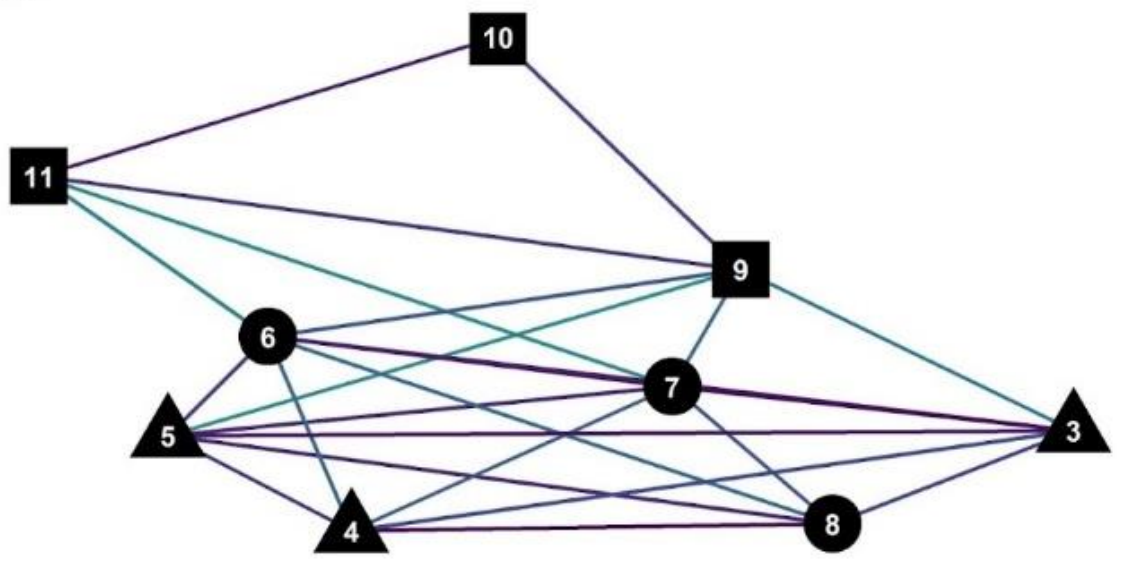

random sample

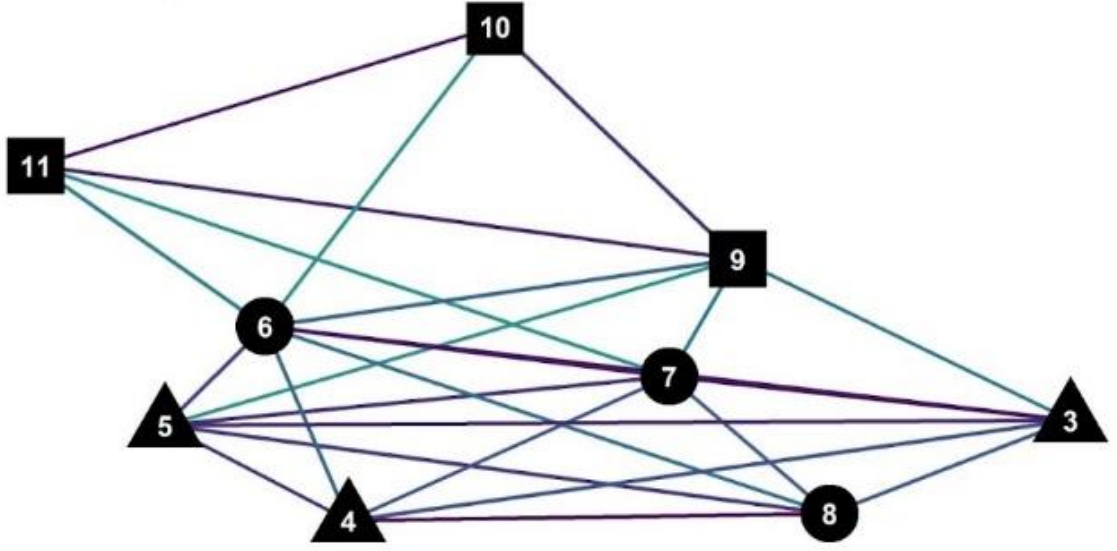

nonrandom sample

10

11

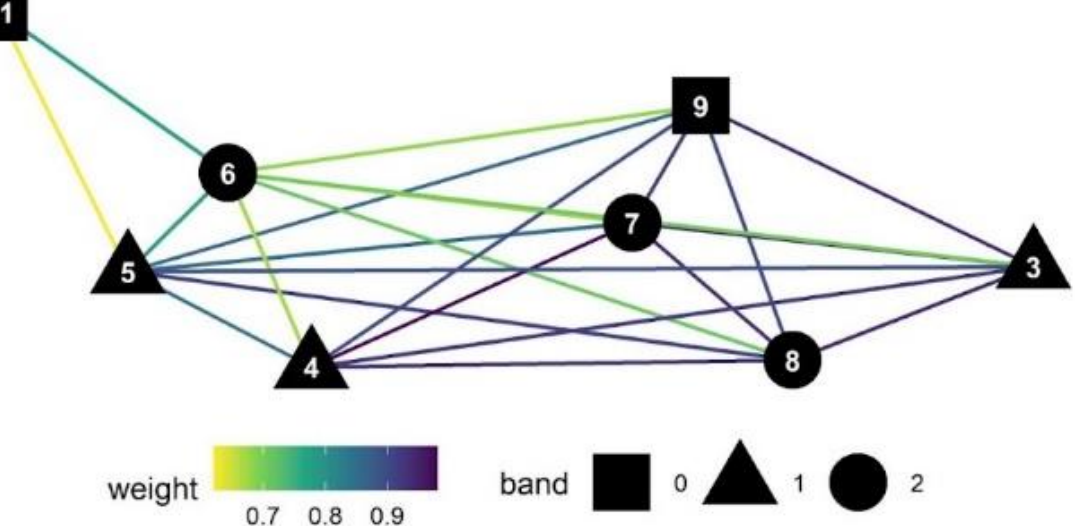

Figure 6. Comparisons of the networks reconstructed from similarities in pot types between camps. The samples are $20 \%$ of the original data. The random sample is nearly identical to the original network. The nonrandom sample has nearly identical connections between each camp, with the exception of camp 7, which has lost all of its ties. Only the strongest $50 \%$ of ties are shown in this graph, and the locations of each node are held constant in the three graphs for easier comparison. 
Table 2. Obsidian Laplacian Distances by Sample Size and Type

\begin{tabular}{lllll}
\hline sample proportion & sample size & random mean & nonrandom mean & p-value \\
\hline $25.0 \%$ & 34 & 7.58 & 9.72 & 0 \\
$50.0 \%$ & 59 & 5.43 & 7.98 & 0 \\
$75.0 \%$ & 85 & 4.81 & 7.24 & 0 \\
\hline
\end{tabular}

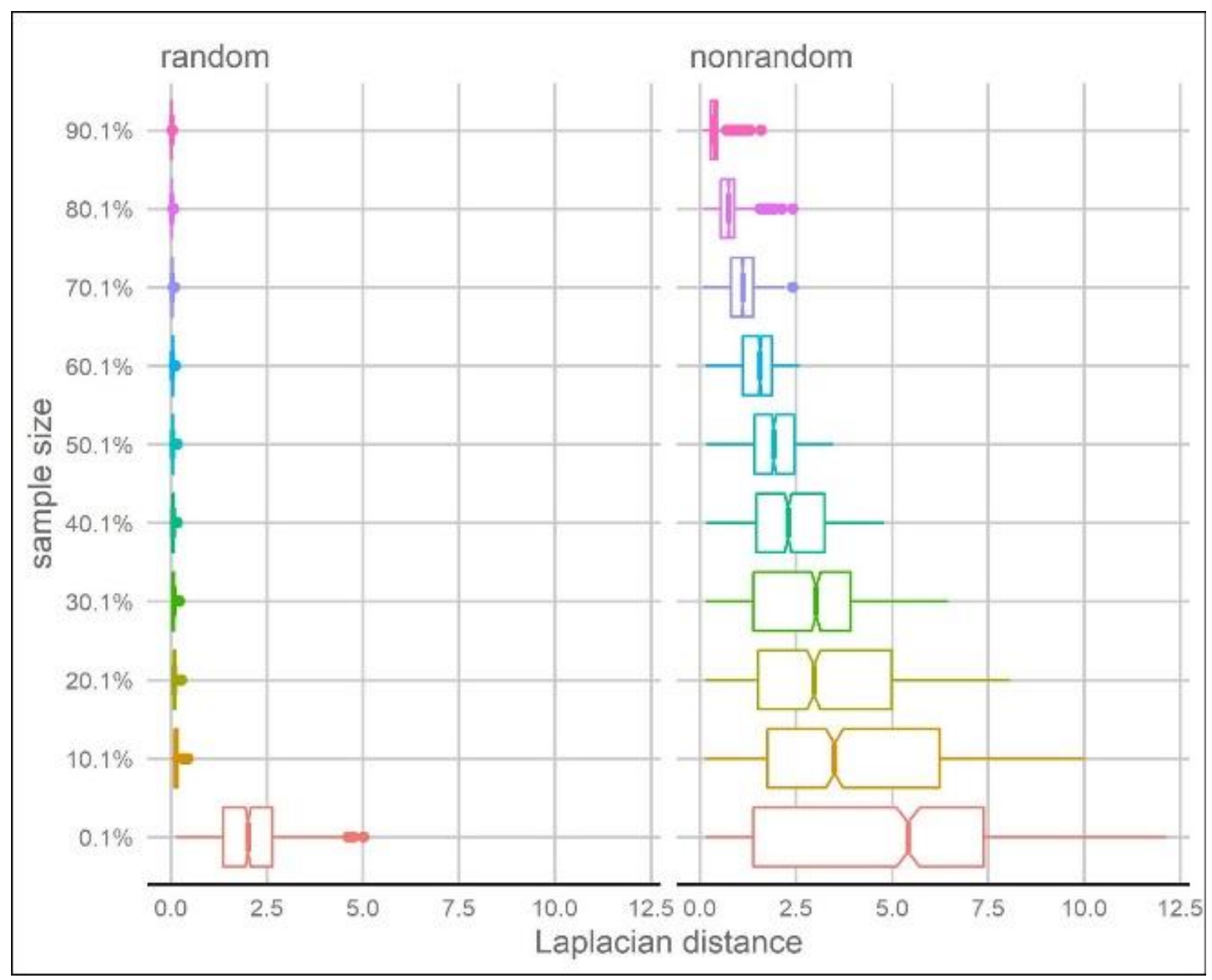

Figure 7. Boxplot comparison of the Laplacian distance between sampled ArchMatNet data for random and nonrandom samples. Notches represent 95\% confidence intervals. 

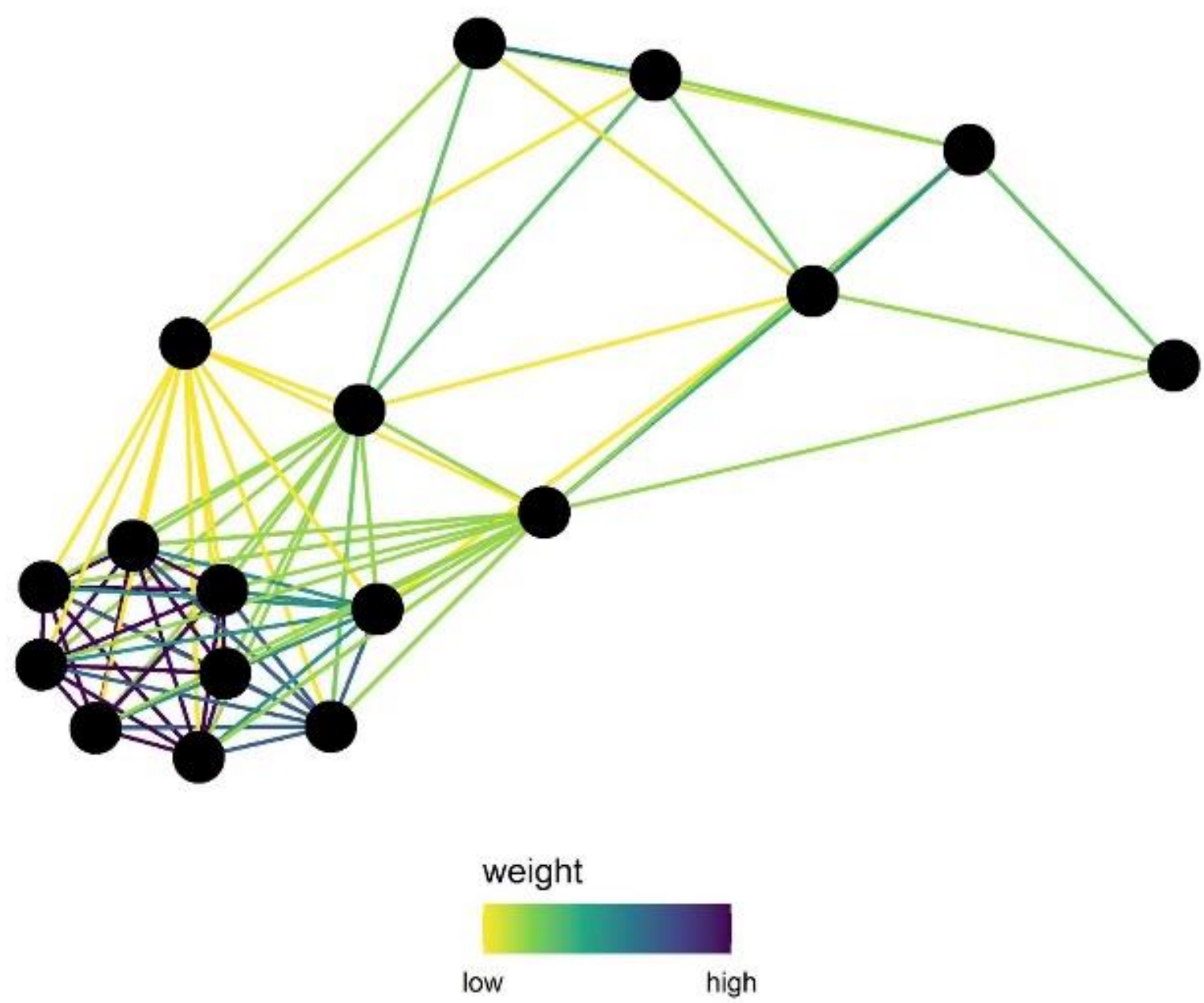

Figure 8. Network graph displaying each archaeology site in the obsidian data. Connections between nodes represent the weighted Jaccard similarity between the obsidian types at each site. Only the strongest $50 \%$ of connections are shown in the graph.

\section{6.b Obsidian Artifacts}

The results of the comparisons between the random and nonrandom sampling of the archaeological data differ from the results presented above. This may be for multiple reasons that are discussed below.

Figure 8 shows the network recreated from the complete Roosevelt obsidian data connected using the weighted Jaccard distance between each site. About half of the sites are densely connected, but all of the sites form a single component of the networkmeaning that every node in the network is connected.

In terms of the impact of sample size on network reconstructions, the Laplacian distances calculated between reconstructed networks show a similar pattern to the ArchMatNet data, in that the larger the sample, the more accurate the network (see Figure 9). However, with the obsidian data, large and medium random samples are not as accurate as they were in the simulated dataset. Even the $75 \%$ random sample size produced much 


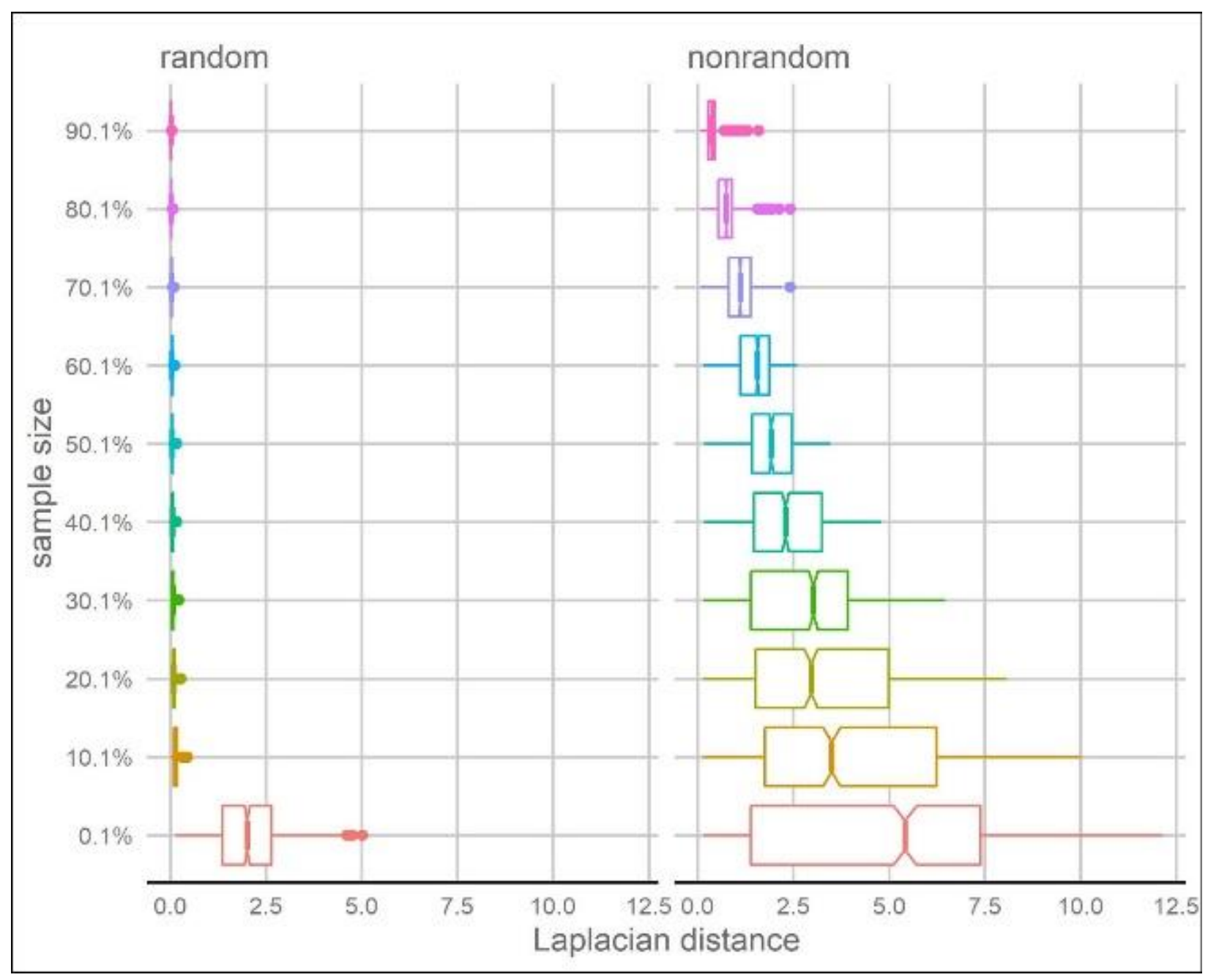

Figure 9. Boxplot comparison of the Laplacian distance between sampled obsidian data for random and nonrandom samples. Notches represent $95 \%$ confidence intervals (outliers with a greater than 20 Laplacian distance are not shown).

more variability than expected. This may be because of the small size of the full dataset, which leads to small sample sizes even when most of the dataset is sampled. In turn, small samples usually tend to show more differences from their population. Nonrandom sample Laplacian distances were still significantly larger than those for random samples (Table 2; $p$-values are calculated using the method described for Table 1). However, it is interesting that, with the obsidian dataset, the Laplacian distances from random and nonrandom samples of the same sizes are more similar than they were for the simulated data.
The results presented here seem to suggest that, with small sample sizes, the effect of random vs nonrandom samples may not be as big as we would have thought. However, that is not to say that biases do not matter here, as the networks reconstructed from the random samples still have far fewer outliers and are on average much more similar to the original network than the networks reconstructed from nonrandom samples. However, the variability shown in the random sample suggests that this data has significant variability in general and may not produce reliable results even if no bias is present in the data. Or it may show 
that the dataset is already biased, which is most likely the case.

\section{Discussion and Conclusion}

The results of this experiment with simulated and archaeological data shows that nonrandom samples are not as adequate to reconstruct networks as random samples. We found that samples as small as $10 \%$ provided accurate network reconstructions, echoing the findings of Peeples and colleagues (2016). Although this requires a large original dataset as the obsidian analysis performed poorly at smaller sample sizes. Moreover, the size of the disparity between the networks reconstructed from both sample types means that researchers need to address sampling biases in any dataset used for network analysis, when possible. All archaeological datasets have some biases. Unfortunately, it can be hard to identify precisely the extent of biases linked to each dataset, given the many unknown variables that affect archaeological assemblages. This research shows that archaeologists must use their best judgement and carefully consider potential variables rather than assume that their dataset is unbiased and thus represents well all facets of the population they are studying. In particular, data with an unknown history or data from small samples might provide exceptionally misleading results. Finally, the results of this research show that while collecting as much data as possible might be a fair attempt to drown out biases, even large datasets can lead to inaccurate results if the dataset is biased.

While this might seem bleak for archaeologists, this is not to say that biased samples_and arguably all archaeological datasets-are too problematic to provide valuable information. For one thing, the results of the research show that, while we cannot trust the absence of links between sites, or their strength, in a biased sample, we can trust their presence. Additionally, we can trust that the co-occurrence of objects is a likely indicator of some form of social contact-be it direct or indirect. This is similar to the results of Costenbader and Valente (2003) who also found that co-occurrence is less affected by subsampling than similarity measures between sites. As argued by Drennan (2009), we can also confidently compare multiple biased samples when their bias is equivalent, or if the known bias can be reasonably accounted for in the results.

With many pre-modern archaeological samples, it can be difficult to determine the extent of bias or even the type of bias present in a dataset. Exploring this question is still a research topic in development, but a few methods have been put forward in the past decade. One way to detect biased samples is by comparing samples with good context to samples with poor or unknown context (Huster 2013). If the datasets from several, largely unbiased samples are similar to datasets where the bias is not well-known, then it is probable, but not certain, that the unknown datasets are not significantly biased. This requires some expertise in the datasets to ensure they represent the same general population and time period.

Random resampling of the datasets can also help detect biases. Here, we demonstrated that even random sampling of the obsidian data produced networks that varied significantly from the complete dataset, which suggests that the complete dataset is biased already. In turn, this shows that the network one could reconstruct from that dataset is not robust and should be treated with caution. Thus, our research shows that similar resampling of any dataset might be worthwhile to assess 
how biased the dataset is and put interpretations into perspective.

The ArchMatNet model provides ideal data to test network reconstructions and explore ways to improve how we can reconstruct them from biased data. Future iterations of the model could introduce different types of bias to explore their impact on the assemblages. These data could then be used not only to better understand how different biased samples affect archaeological data and network reconstructions, but also to find solutions to mitigate the effects of biased samples on those reconstructions.

Acknowledgements: The authors thank the organizers of the CAA 2021 conference and the "New challenges in archaeological network research" session for the opportunity to first present this research. We also thank Matthew Peeples for his contributions.

Data Availability: The ArchMatNet model can be found here: https://www.comses.net/codebaserelease/e7ed1aec-af7c-42c8-a7fd15469f6d4067/. The R code and data used in this analysis can be found here: https://doi.org/10.17605/OSF.IO/J36EB.

\section{References}

Birch, J and Hart, J P 2018 Social Networks and Northern Iroquoian Confederacy Dynamics, American Antiquity, 83(1): 13-33. DOI: https://doi.org/10.1017/aaq.2017.59.

Bolland, J M 1988 Sorting out centrality: An analysis of the performance of four centrality models in real and simulated networks, Social networks, 10(3): 233253. DOI: https://doi.org/10.1016/03788733(88)90014-7.
Borgatti, S P, Carley, K M and Krackhardt, D 2006 On the robustness of centrality measures under conditions of imperfect data, Social networks, 28(2): 124-136. DOI: https://doi.org/10.1016/j.socnet.2005.05. 001.

Boyd, R and Richerson, P J 1985 Culture and the evolutionary process. University of Chicago Press.

Brandes, U, Robins, G, McCranie, A and Wasserman, $S 2013$ What is Network Science?, Network Science, 1(1): 1-15. DOI: https://doi.org/10.1017/nws.2013.2.

Brughmans, T 2013 Thinking Through Networks: A Review of Formal Network Methods in Archaeology, Journal of Archaeological Method and Theory, 20(4): 623-662. DOI:

https://doi.org/10.1007/s10816-0129133-8.

Brughmans, T and Peeples, M 2017 Trends in Archaeological Network Research: A Bibliometric Analysis, Journal of Historical Network Research, 1(1): 1-24. DOI: https://doi.org/10.25517/jhnr.v1i1.10.

Chung, F R K 1997 Spectral Graph Theory. American Mathematical Soc.

Clarke, D 1973 Archaeology: the loss of innocence, Antiquity, 47(185): 6-18.

DOI:

https://doi.org/10.1017/S0003598X0003 461X.

Collar, A, Coward, F, Brughmans, T and Mills, B J 2015 Networks in

Archaeology: Phenomena, Abstraction, Representation, Journal of Archaeological Method and Theory, 22(1): 1-32. DOI:

https://doi.org/10.1007/s10816-0149235-6. 
Costenbader, E and Valente, T W 2003 The stability of centrality measures when networks are sampled, Social networks, 25(4): 283-307. DOI: https://doi.org/10.1016/S03788733(03)00012-1.

Drennan, R D 2009 Statistics for Archaeologists: A Common Sense Approach. Dordrecht: Springer.

Galaskiewicz, J 1991 Estimating point centrality using different network sampling techniques, Social networks, 13(4): 347-386. DOI: https://doi.org/10.1016/03788733(91)90002-B.

Gjesfjeld, E 2015 Network Analysis of Archaeological Data from HunterGatherers: Methodological Problems and Potential Solutions, Journal of Archaeological Method and Theory, 22(1): 182-205. DOI:

https://doi.org/10.1007/s10816-0149232-9.

Golitko, M, Meierhof, J, Feinman, G M and Williams, P R 2012 Complexities of Collapse: The Evidence of Maya Obsidian as Revealed by Social Network Graphical Analysis, Antiquity, 86: 507-523.

Gravel-Miguel, C and Coward, F n.d. Palaeolithic Social Networks and Behavioural Modernity. In: Peeples, M.A., Munson, J., Mills, B., and Brughmans, T. (eds.) The Oxford Handbook of Archaeological Network Research. Oxford: Oxford University Press.

Greenbaum, G, Friesem, D E, Hovers, E, Feldman, M W and Kolodny, O 2019 Was inter-population connectivity of Neanderthals and modern humans the driver of the Upper Paleolithic transition rather than its product?, Quaternary science reviews, 217: 316-329. DOI: https://doi.org/10.1016/j.quascirev.2018. 12.011.

Hage, P 1977 Centrality in the Kula Ring, The journal of the Polynesian Society., 86(1): 27-36.

Hage, P, Harary, F and Others 1991 Exchange in Oceania: a graph theoretic analysis. Oxford University Press.

Hunt, T L 1988 Graph theoretic network models for Lapita exchange: a trial application. In: Kirch, P.V. and Hunt, T.L. (eds.) Archaeology of the Lapita cultural complex: a critical review. No. 5 . Seattle: Thomas Burke Memorial Washington State Museum Research Reports. pp. 135-155.

Huster, A C 2013 Assessing systematic bias in museum collections: a case study of spindle whorls, Advances in Archaeological Practice, 1(2): 77-90.

Irwin, G 1978 Pots and entrepots: a study of settlement, trade and the development of economic specialization in Papuan prehistory, World Archaeology, 9(3): 299-319.

Janssen, M A, Alessa, L N, Barton, M, Bergin, S and Lee, A 2008 Towards a Community Framework for Agent-Based Modelling', Journal of Artificial Societies and Social Simulation, 11(2):

Knappett, C 2008 The Neglected Networks of Material Agency: Artefacts, Pictures and Texts. In: Knappett, C. and Malafouris, L. (eds.) Material Agency:

Towards a Non-Anthropocentric Approach. Boston, MA: Springer US. pp. 139-156. DOI: https://doi.org/10.1007/978-0-38774711-8_8.

Lee, S, Yook, S-H and Kim, Y 2009 Centrality measure of complex networks using biased random walks, The European physical journal. B, 68(2): 
277-281. DOI:

https://doi.org/10.1140/epjb/e200900095-5.

Mackay, A, Stewart, B A and Chase, B M 2014 Coalescence and fragmentation in the late Pleistocene archaeology of southernmost Africa, Journal of Human Evolution, 72: 26-51. DOI: https://doi.org/10.1016/j.jhevol.2014.03. 003.

Marczewski, E and Steinhaus, H 1958 On a certain distance of sets and the corresponding distance of functions, Colloquium Mathematicum, 6(1): 319327.

McBrearty, S and Brooks, A S 2000 The Revolution that wasn't: a New Interpretation of the Origin of Modern Human Behavior, Journal of human evolution, 39: 453-563.

Migliano, A B, Battiston, F, Viguier, S, Page, A E, Dyble, M, Schlaepfer, R, Smith, D, Astete, L, Ngales, M, GomezGardenes, J, Latora, V and Vinicius, L 2020 Hunter-gatherer multilevel sociality accelerates cumulative cultural evolution, Science Advances, 6(9): eaax5913. DOI:

https://doi.org/10.1126/sciadv.aax5913.

Mills, B J 2017 Social Network Analysis in Archaeology, Annual Review of Anthropology, 46(1): 379-397. DOI: https://doi.org/10.1146/annurev-anthro102116-041423.

Mills, B J, Clark, J J, Peeples, M A, Haas, W R, Roberts, J M, Hill, J B, Huntley, D L, Borck, L, Breiger, R L, Clauset, A and Shackley, M S 2013 Transformation of social networks in the late pre-Hispanic US Southwest, Proceedings of the National Academy of Sciences of the United States of America, 110(15): 5785-5790. DOI: https://doi.org/10.1073/pnas.121996611 0 .
Peeples, M A 2019 Finding a Place for Networks in Archaeology, Journal of Archaeological Research, 1-49. DOI: https://doi.org/10.1007/s10814-01909127-8.

Peeples, M A and Haas, W R 2013 Brokerage and Social Capital in the Prehispanic U.S. Southwest, American anthropologist, 115(2): 232-247. DOI: https://doi.org/10.1111/aman.12006.

Peeples, M A, Mills, B J, Haas, W R J, Clark, J J and Roberts, J M J 2016 Analytical Challenges for the Application of Social Network Analysis in Archaeology. In: Brughmans, T., Collar, A., and Coward, F. (eds.) The Connected Past: Challenges to Network Studies in Archaeology and History. Oxford: Oxford University Press. pp. 59-84.

R Core Team 2021 R: A Language and Environment for Statistical Computing. $R$ Foundation for Statistical Computing, Vienna, Austria.

Rice, G ed. 1998 A Synthesis of Tonto Basin Prehistory: The Roosevelt Archaeology Studies, 1989 to 1998. Roosevelt Monograph Series 10 Anthropological Field Studies 40. Tempe: Arizona State University, Office of Cultural Resource Management, Dept. of Anthropology.

Rivera-Hutinel, A, Bustamante, R O, Marín, V H and Medel, R 2012 Effects of sampling completeness on the structure of plant-pollinator networks, Ecology, 93(7): 1593-1603. DOI: https://doi.org/10.1890/11-1803.1.

Roosevelt Platform Mound Study (RPMS) 2014 Access Database (tDAR id: 427111). DOI: https://doi.org/10.6067/XCV8TQ63KK.

Shimada, Y, Hirata, Y, Ikeguchi, T and Aihara, K 2016 Graph distance for 
complex networks, Scientific reports, 6 : 34944. DOI:

https://doi.org/10.1038/srep34944.

Stiner, M and Kuhn, S 1992

Subsistence, Technology, and Adaptive Variation in Middle Paleolithic Italy, American Anthropologist, 94: 306-338.

Tantardini, M, leva, F, Tajoli, L and Piccardi, C 2019 Comparing methods for comparing networks, Scientific reports, 9(1): 17557. DOI: https://doi.org/10.1038/s41598-01953708-y.

Terrell, J 1976 Island Biogeography and Man in Melanesia, Archaeology \& Physical Anthropology in Oceania, 11(1): 1-17.

Terrell, J E 1977 Human biogeography in the Solomon Islands, Fieldiana.

Anthropology, 68(1): 1-47.

Terrell, J E 2010 Language and Material Culture on the Sepik Coast of Papua New Guinea: Using Social Network Analysis to Simulate, Graph, Identify, and Analyze Social and Cultural Boundaries Between Communities, The Journal of Island and Coastal Archaeology, 5(1): 3-32.

Wills, P and Meyer, F G 2020 Metrics for graph comparison: A practitioner's guide, PLoS One, 15(2): e0228728.

DOI:

https://doi.org/10.1371/journal.pone.022 8728.

You, K 2020 NetworkDistance: Distance Measures for Networks. 